\title{
Increasing targeting scope of adenosine base editors in mouse and rat embryos through fusion of TadA deaminase with Cas 9 variants
}

\section{Dear Editor,}

The clustered regularly interspaced short palindromic repeat (CRISPR) system has been widely adapted to genome editing to either introduce or correct genetic mutations (Wang et al., 2016). However, due to competition with the dominant non-homologous end-joining (NHEJ) pathway, precise genome modifications through Cas9-stimulated homologous recombination $(\mathrm{HR})$ is inefficient. Through fusion of cytidine deaminases, APOBEC1 (apolipoprotein B editing complex 1) or AID (activation-induced deaminase), with Cas9 variants, several groups have developed the cytidine base editor (BE) systems (Komor et al., 2016; Li et al., 2018; Nishida et al., 2016). The BE system achieves programmable conversion of $C \cdot G$ base pairs to $T \cdot A$ without double-stranded DNA cleavage (Zhou et al., 2017). More recently, adenine base editors (ABEs), which efficiently convert $A \cdot T$ base pairs to $G \cdot C$ in genomic DNA, have been developed via fusion of an engineered tRNA adenosine deaminase (ecTadA from Escherichia coli) with nCas9 (Gaudelli et al., 2017). The ABE system has quickly been adapted to generate disease models and correction of genetic disease in mice (Ryu et al., 2017; Liu et al., 2018). However, whether the editing efficiency and the targeting scope of ABE could be improved is largely unexplored. In this study, we describe the efficient generation of base-edited mice and rats modeling human diseases through $A B E s$ with highest efficiency up to $100 \%$. We also demonstrate an increase of $A B E$ activity through injection of chemically modified tracrRNA and crRNA in mouse zygotes, and the expansion of editing scope by fusion of an ecTadA mutant to SaCas9n-KKH and Cas9n-VQR variants in both cells and embryos. Our study suggests that the ABE system is a powerful and convenient tool to introduce precise base conversions in rodents.

To test the $A B E$ efficiency in embryos, we injected $A B E$ mRNA (Fig. 1A) together with sgRNA targeting the TATA box of the $H b b$-bs gene, into C57BL6 strain mouse zygotes (Fig. S1A and Table S1). Overlapping A/G peaks in the target sites were identified in 14/27 of F0 mice as determined by the chromatograms of Sanger sequencing (Figs. $2 \mathrm{~F}$ and S1B). Further analysis by deep sequencing revealed allelic frequencies from $6 \%-71 \%$ among the founders (Fig. S1C). In individual allele, the editing window was extended from position $A_{2}-A_{9}$ in mouse embryos, which is broader than the window spanning position $A_{4}-A_{7}$ observed in mammalian cell lines (Gaudelli et al., 2017) (Fig. S1B and S1C). These data demonstrate that $A B E$ is efficient to generate point mutant mice and its mutation window expands in embryos.

Next, we tested the capability of $A B E$ to precisely mutate $A: T$ pairs for disrupting the stop codon of the gene encoding the fumarylacetoacetate hydrolase (Fah) (Fig. 1B), whose mutations cause hereditary tyrosinemia type I (HTI) in humans. We observed high $A>G$ conversion efficiency (39/47) among F0 mice with allelic frequencies varying from $7 \%-99 \%$ as determined by deep sequencing (Figs. 1B, 1C, $2 \mathrm{~F}$ and $\mathrm{S} 2 \mathrm{~A}$ ). Increasing the sgRNA concentration from $50 \mathrm{ng} / \mu \mathrm{L}$ to $100 \mathrm{ng} / \mu \mathrm{L}$ results in $100 \%$ (13/13) point mutation rate in F0 mice (Figs. $2 \mathrm{~F}$ and S2B). Since disruption of the stop codon usually affects mRNA stability and protein expression (Frischmeyer et al., 2002), the Fah mRNA and protein levels were dramatically impaired (Fig. S2C and S2D). Through immunohistochemistry analysis of the liver tissue from founder F0-F32, Fah protein expression was almost undetectable (Fig. 1D) suggesting this founder was a homozygote (Fig. 1B and 1C). To investigate germline transmission efficiency, founder mice were crossed with wild type or with other founders. We observed high germline transmission efficiency (Fig. S3A and S3B). In homozygous F1 mice, the expression of Fah mRNA and protein was lost (Fig. S3C-E). After withdrawal of the 2-(2-nitro-4-trifluoromethylbenzoyl)-1,3-cyclohexanedione (NTBC) treatment, the phenotypes of Fah mutant homozygotes were similar to previous HTI model, including loss of body weight and perturbation of serum biomarkers (Shao et al., 2018) (Fig. S3F and $S 3 G$ ). As mutations that generate premature stop codons are common drivers in various genetic diseases (Keeling et al., 2014), ABE has a promising potential for readthrough of premature stop codons in certain genetic diseases as demonstrated in the mouse DMD model (Ryu et al., 2017).

Previous study demonstrated that 2'-O-methyl-3'-phosphorothioate (MS) modification on each ends of RNA can increase its stability, thus enhancing the Cas9 genome 
A

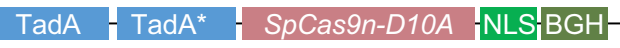

B

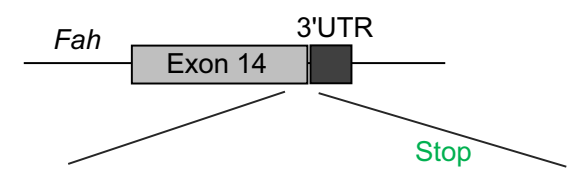

WT CCTGCCCTTTCACCAGCC TGAAG CTCCGGA $\hat{5}$

F0-F10 CCTGCCCTTTCACCAGCC cGAAG CTCCGGA 77.6 (Stop>R) CCTGCCCTTTCACCAGCCTGAAG CTCCGGA 22.3 (WT)

F0-F19 CCTGCCCTTTCACCAGCC cGAAG CTCCGGA 49.6 (Stop>R) CCTGCCCTTTCACCAGCCTGAAG CTCCGGA 50.3 (WT)

F0-F21 CCTGCCCTTTCACCAGCC cGAAG CTCCGGA 77 (Stop>R) CCTGCCCTTTCACCAGCCTGAAG CTCCGGA 23 (WT)

F0-F24 CCTGCCCTTTCACCAGCC cGAAG CTCCGGA 7.6 (Stop>R) CCTGCCCTTTCACCAGCCTGAAG CTCCGGA 92.4 (WT) F0-F32 CCTGCCCTTTCACCAGCC cGAAG CTCCGGA 99 (Stop>R)
C

WT C C TG C C C T T T CAC CA G C C $\frac{T G A A G}{\text { Stop }}$

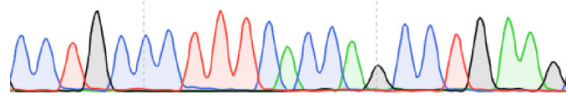

E

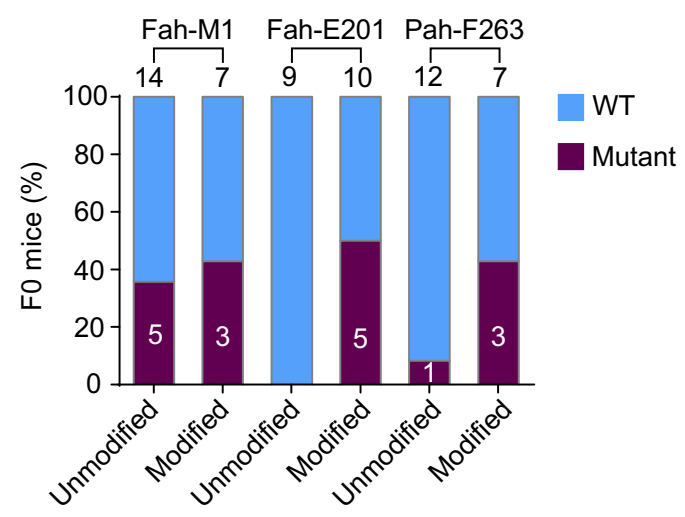

G

WT GCA $\frac{G A C}{A s p} \frac{A T C}{\| e} T G C G G C T T C$ C A GGG

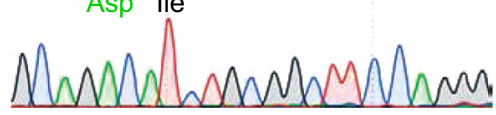

F0-G22 GCAGGCGTCTGCGGCTTC CAGGG

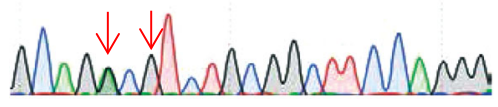

F0-G26 GCAGACGTCTGCGGCTTC CA GGG

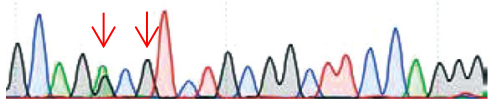

Frequency $(\%)$

F

D

F0-F32 C C TG C C C T T T CAC CA G C C $\underline{\operatorname{Arg} A}$

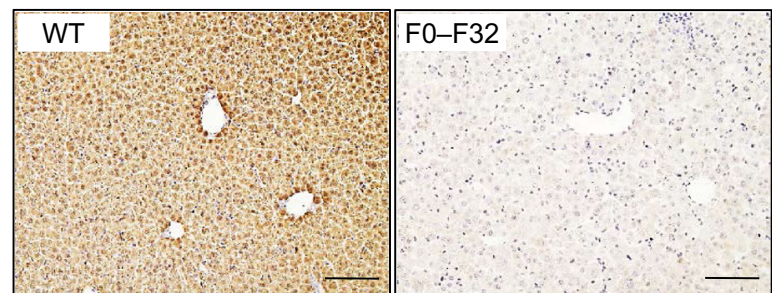

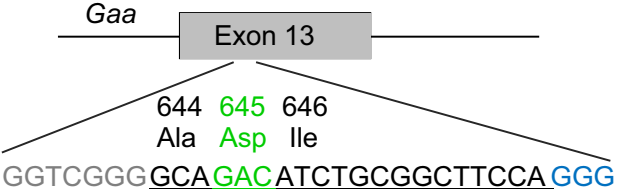

$$
\begin{array}{llll}
\mathbf{3} & \mathbf{5} & \mathbf{4}
\end{array}
$$

Frequency (\%)

F0-G3 GGTCGGG GCAGACg TCTGCGGCTTCCA GGG 48.7 (I646V) GGTCGGG GCg Gg g TCTGCGGCTTCCA GGG 21.6 (D645G, I646V) GGTCGGG GCAGg Cg TCTGCGGCTTCCA GGG 18.6 (D645G, I646V) GGTCGGG GCAGACATCTGCGGCTTCCA GGG 10.7 (WT)

F0-G19 GGTCGGG GCAGACg TCTGCGGCTTCCA GGG 18.4 (I646V) GGTCGGG GCg GACgTCTGCGGCTTCCA GGG 28 (I646V) GGTCGGG GCAGg CATCTGCGGCTTCCA GGG 15.4 (D645G) GGTCGGG GCg ggCg TCTGCGGCTTCCA GGG 4.1 (D645G, I646V) GGTCGGG GCAGACATCTGCGGCTTCCA GGG 33.3 (WT)

F0-G22 GGTCGGG GCAGACg TCTGCGGCTTCCA GGG 49.2 (I646V) GGTCGGG GCAGg Cg TCTGCGGCTTCCA GGG 50 (D645G, I646V)

F0-G26 GGTCGGG GCAGACg TCTGCGGCTTCCA GGG 62.5 (I646V) GGTCGGG GCAGg g TCTGCGGCTTCCA GGG 37.1 (D645G, I646V)

$\mathrm{H}$

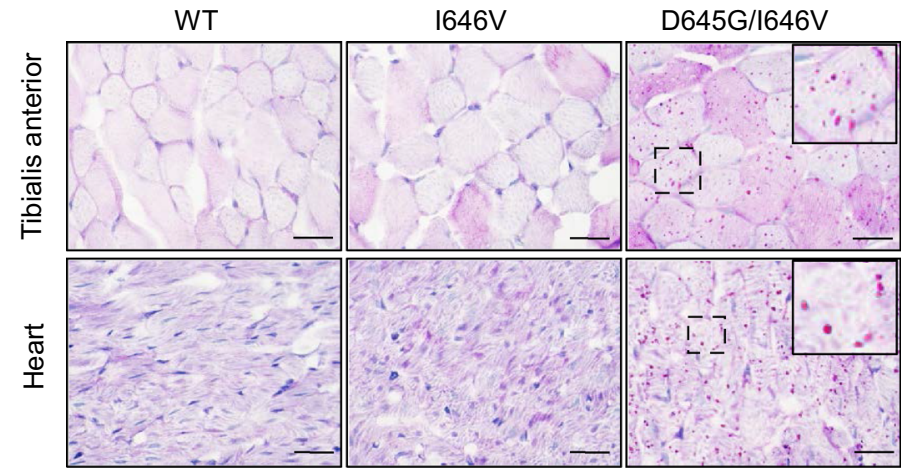


Figure 1. $A B E$ induces efficient $A>G$ conversion in mouse and rat embryos. (A) $A$ schematic view of the $A B E 7.10$ vector used as the template for ABE mRNA transcription. (B) $A$ schematic view of the target site at the Fah stop codon. Target sequence is underlined. PAM sequence is labeled in blue. Stop codon is labeled in green. Arrow head indicates the targeted thymine. Base substitutions are labeled in red. Allele frequencies are listed to the right. (C) Sanger sequencing chromatograms from the WT and F0-F32 founder. T>C conversion is indicated by the red arrow. (D) IHC staining of the liver tissue sections from WT and F0-F32 founder by anti-Fah antibody. Scale bar, $100 \mu \mathrm{m}$. (E) The editing efficiencies at three different target sites with chemically modified crRNA/tracrRNAs or unmodified sgRNAs. The numbers indicate the number of pups generated. (F) A schematic view of the target site in exon 13 of the rat Gaa gene and deep sequencing results from the genomic DNA of the mutant founders. PAM sequence is labeled in blue. Target sequence is underlined with codon 644,645 and 646 indicated by their amino acid. Base substitutions are labeled in red. Allele frequencies are listed to the right. (G) Sanger sequencing chromatograms from the genomic DNA of WT and two mutant F0 founders. Double peak signals caused by $A>G$ conversions are indicated by red arrows. Codon 645 and 646 of WT and mutant alleles are underlined. (H) PAS staining of heart and Tibialis anterior cryo-sections from 3 week old WT, I646V and D645G/1646V homozygotes. Scale bar, $20 \mu \mathrm{m}$.

editing efficiency (Hendel et al., 2015). To explore whether MS modification on RNA also increases ABE activity, MS modified crRNAs and tracrRNAs were directly compared with in vitro transcribed sgRNAs. Indeed, in all three targets tested, the $A B E$ editing efficiencies were higher when injected with MS modified crRNAs and tracrRNAs (Figs. 1E and S4). Moreover, the mutation efficiencies in individual mice were also increased in the group that received chemically modified RNAs (Fig. S4A-D). It suggests that increasing of sgRNA stability is an efficient strategy to increase $A B E$ induced editing in embryos.

To investigate the activity of $A B E$ in rats, we aimed to target the acid alpha-glucosidase (Gaa) gene to mutate aspartic acid (Asp) at codon 645 in exon 13, which is a mutation identified in glycogen storage disease type II (GSDII; Pompe disease) patients (Kroos et al., 2004) (Fig. 1F). GSDII is a fatal disorder characterized by progressive loss of skeletal and/or heart muscle function. Sanger sequencing data suggested that $85 \%(28 / 33)$ of rats carried single or multiple $A>G$ substitutions between position $A_{3}-A_{7}$ in the target leading to $1646 \mathrm{~V}$ or D645G mutations $\left(A_{3}\right.$ is a synonymous mutation) (Figs. $1 F, 1 G$ and $2 F$ ). After deep sequencing of all founders, the editing frequency in individual rats ranged from $26 \%-100 \%$ (Fig. S5A). The founders which had higher mutation rates showed significantly reduced Gaa activity (Fig. S5B). To analyze the phenotype of the Pompe disease rat model, we crossed two pairs of founders and obtained two Gaa mutant rat strains, $1646 \mathrm{~V}$ and
D645G/1646V with an overall germline transmission efficiency of 92\% (12/13) (Fig. S5C). In D645G/1646V homozygous rats, Gaa enzyme activity was $0.2-0.4 \mathrm{nmol} / \mathrm{h} / \mathrm{mg}$ which is $1.2 \%-2.5 \%$ of that in wild-type controls (Fig. S5D) and is similar to the enzyme activity determined in D645 mutant patients (Kroos et al., 2004). However, in I646V rats, Gaa activity was mildly reduced, suggesting that the 646 site might not be a critical site for Gaa since no point mutation at this site has been reported among 558 known mutations in patients (Pompe Mutatiedatabase http://cluster15.erasmusmc.nl/klgn/ pompe/mutations.html). Gaa mutation causes abnormal accumulation of large lysosomes filled with glycogen in multiple tissues which lead to heart failure and skeletal muscle weakness depending on the severity caused by the mutation. Using PAS staining of heart, tibialis anterior and rectus femoris cryo-sections from 3 week-old Gaa mutant rats, we found an accumulation of PAS-positive vacuoles in all the tested tissues in D645G/1646V rats (Figs. $1 \mathrm{H}$ and S5E). These data suggest the successful generation of a Pompe disease rat model.

The PAM restriction of SpCas9-based ABE limits the number of potential targets. To expand the targeting scope of $A B E$, we fused an ecTadA variant with SaCas9n-KKH (PAM: NNNRRT) (Kleinstiver et al., 2015a) or Cas9n-VQR (PAM: NGA) (Kleinstiver et al., 2015b) to generate SaKKH-ABE and VQR-ABE respectively (Fig. 2A). To investigate the editing window and efficiency, 4 targets for either SaKKHABE or VQR-ABE were tested in HEK293 cells. Deep sequencing data showed that both of the $A B E s$ actively generated $A>G$ conversions in cells (Fig. $2 B$ and $2 C$ ). The editing efficiencies were up to $\sim 50 \%$ of both SaKKH-ABE and VQR-ABE variants in certain position (Fig. $2 B$ and $2 C$ ). We noticed that the editing window of SaKKH-ABE was expanded (position $A_{3}-A_{14}$ on EMX1 site 2) compared to $A B E$. Our preliminary data also suggested that the highly active position of SaKKH-ABE in the target was $A_{8}-A_{13}$ which was closer to the PAM sequence compared to $A B E$.

To test whether these two ABEs function in mouse embryos, we injected $m R N A$ of $A B E$ variants with individual sgRNAs. After microinjection of SaKKH-ABE mRNA and sgRNA, $16 \%$ (3 out of 19) of the mice carried a single mutation in the Otc locus with an editing rate ranging from $30 \%-54 \%$ in single founders as determined by deep sequencing (Fig. 2D and 2F). For VQR-ABE, we also directly injected VQR-ABE mRNA and sgRNA targeting $H b b-b s$ into mouse embryos. The editing efficiency was $20 \%$ (6 out of 30 ) at the $H b b$-bs locus with the $A>G$ conversion efficiency ranging from $2 \%-52 \%$ as determined by deep sequencing (Fig. 2E and 2F). These data suggest that expansion of the ABE editing scope through fusion with Cas9 variants is efficient in both cell lines and mouse embryos.

To evaluate the off-target effects of this $A B E$, we predicted the potential off-target sites of sgRNA targeting the Fah stop codon based on sequence similarity through the on-line target prediction program (http://crispr.mit.edu/). 20 predicted off-target sites of 3 highly edited founders for each sgRNA 
A

- TadA - TadA* SaCas9n-KKH -NLS-BGH-

- TadA - TadA* - SpCas9n-VQR -NLS-BGH -

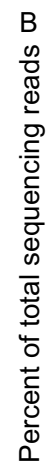

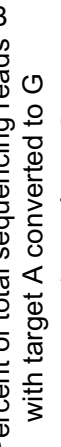

C

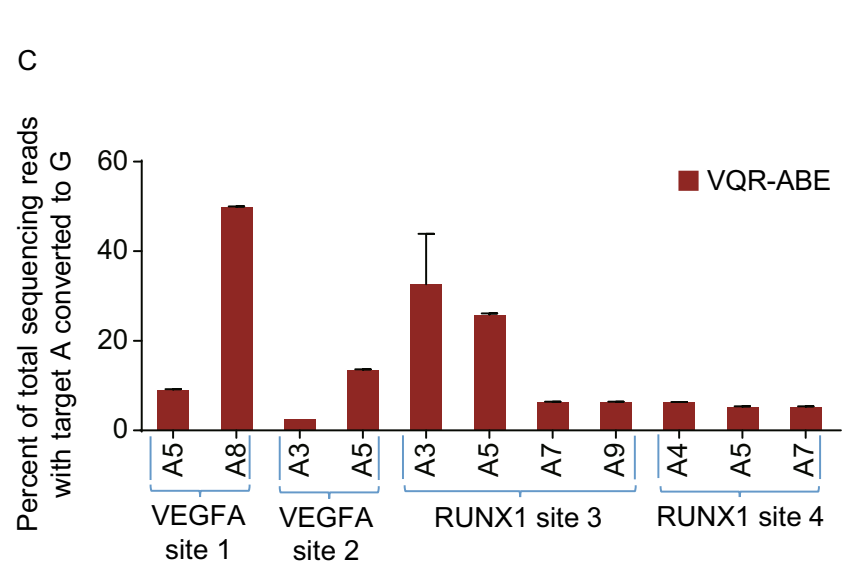

$\mathrm{F}$

F
D

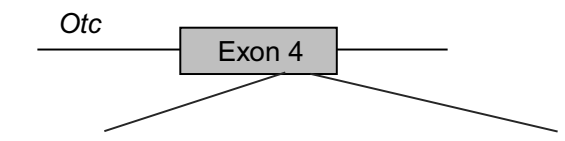

WT CTTACCA CACAAGACATTCACTTGGGTGTGAAT

9 $\quad$ Frequency $(\%)$

F0-O8 CTTACCACACAAGAC gTTCACTTGGGT GTGAAT 54.6 CTTACCACACAAGACATTCACTTGGGT GTGAAT 44.7

F0-09 CTTACCACACAAGAC gTTCACTTGGGT GTGAAT 31.6 CTTACCACACAAGACATTCACTTGGGT GTGAAT 68.2

F0-010 CTTACCACACAAGAC gTTCACTTGGGT GTGAAT 30.5

EMX1 site 1 EMX1 site $2 \quad$ RUNX1 site 1 Hek293 site 1

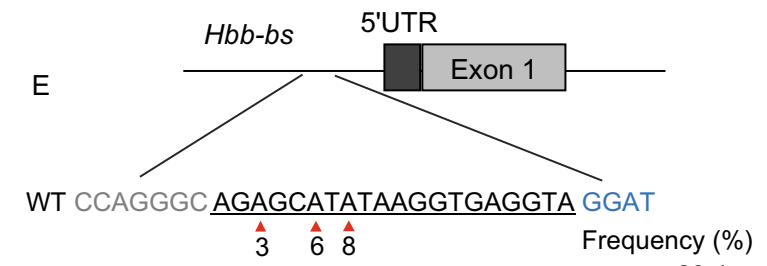

F0-b4 CCAGGGC AGAGC gTATAAGGTGAGGTAGGAT 23.4 CCAGGGC AGAGCATATAAGGTGAGGTA GGAT 72.2

F0-b5 CCAGGGC AGAGC gTATAAGGTGAGGTAGGAT 36.7 CCAGGGC AGAGCATATAAGGTGAGGTAGGAT 58

F0-b10 CCAGGGC AGAGCgTgTAAGGTGAGGTAGGAT 17.9 CCAGGGC AGAGCAT gTAAGGTGAGGTA GGAT 17 CCAGGGC AGAGCATATAAGGTGAGGTA GGAT 59.9 F0-b12 CCAGGGC AGAGCgTgTAAGGTGAGGTAGGAT 52.1 CCAGGGC AGAGCATATAAGGTGAGGTA GGAT 41.4

F0-b13 CCAGGGC AGgGC gTATAAGGTGAGGTAGGAT 2.2 CCAGGGC AGAGCATATAAGGTGAGGTAGGAT 91.1

F0-b14 CCAGGGC AGAGCgTgTAAGGTGAGGTAGGAT 3 CCAGGGC AGAGCATATAAGGTGAGGTAGGAT 90.1

\begin{tabular}{|c|c|c|c|c|c|}
\hline Strain & Gene & Target site & $\begin{array}{l}\text { mRNA/sgRNA } \\
(\mathrm{ng} / \mu \mathrm{L})\end{array}$ & $\begin{array}{l}\text { Injected/Transplanted } \\
\text { embryos }\end{array}$ & No. of mutants/offspring \\
\hline \multirow{5}{*}{ C57/BL6 } & $H b b-b s$ & TATA box & $50 / 100$ & $190 / 190$ & $14 / 27(52)$ \\
\hline & \multirow{2}{*}{$F a h$} & Stop codon & $50 / 50$ & $105 / 104$ & $39 / 47(83)$ \\
\hline & & *Stop codon & $50 / 100$ & $46 / 45$ & $13 / 13(100)$ \\
\hline & ${ }^{* *} \mathrm{Otc}$ & Exon 4 & $50 / 50$ & $47 / 45$ & $3 / 19(16)$ \\
\hline & ${ }^{* * *} H b b-b s$ & TATA box & $50 / 50$ & $78 / 75$ & $6 / 30(20)$ \\
\hline Sprague-Dawley & Gaa & D645 & $50 / 100$ & $104 / 102$ & $28 / 33(85)$ \\
\hline
\end{tabular}

* Increased sgRNA concentration to $100 \mathrm{ng} / \mu \mathrm{L}$ for microinjection.

** SaKKH-ABE mRNA is used instead of ABE mRNA.

${ }^{* * *}$ VQR-ABE mRNA is used instead of ABE mRNA. 
Figure 2. Fusion of the adenosine deaminase with Cas9 variants. (A) A schematic view of SaKKH-ABE and VQR-ABE vectors. (B) Frequencies of $A>G$ conversions induced by SaKKH-ABE in HEK293T cell line. (C) Frequencies of $A>G$ conversions induced by VQR-ABE in HEK293T cell line. (D) Genotypes of the founders produced by the SaKKH-ABE system in the Otc locus. Target sequence is underlined. Arrow head indicates the targeted thymine. PAM sequence is labeled in blue. Base substitutions are labeled in red. Allele frequencies are listed to the right. (E) Genotypes of the founders produced by the VQR-ABE system in the $H b b$ - $b$ s locus. Target sequence is underlined. Arrow head indicates the targeted thymine. PAM sequence is labeled in blue. Base substitutions are labeled in red. Allele frequencies are listed to the right. $(F)$ Summary of the targeted sites and injection parameters used to generate the point mutant rodents in the study.

were selected, and PCR products were amplified and subjected to deep sequencing. We found that the frequency of off-target mutation was below $0.2 \%$ (due to the threshold of $\mathrm{Hi}$-Tom method) which is similar to wild-type controls by analyzing a total of $50,000-100,000$ reads/site via the web site (http://www.hi-tom.net/hi-tom/), demonstrating that $A B E$ might have very few or no off-target effects at these tested sites (Fig. S6). It suggests that $A B E$ is an accurate base editing tools for generation of mouse and rat point mutant strains.

In summary, we demonstrated that $A B E$ and its variants efficiently generate site-specific $A: T>G: C$ conversions in cell lines, mouse and rat embryos. We found that the editing window of $A B E 7.10$ in rodent embryos is from position 2-9. To the best of our knowledge, this is the first report to demonstrate efficient generation of point mutations through base editors in rats. The SaKKH-ABE and VQR-ABE system will be important tools to diversify the range of $A B E$ targets in the genome. As $A>G$ conversion may correct $48 \%$ of the pathogenic human SNPs (Gaudelli et al., 2017), in combination with $\mathrm{BEs}$, these base editing systems have promising potential not only for generation of disease models, but more importantly for therapy of hereditary diseases caused by point substitutions.

\section{FOOTNOTES}

This work was partially supported by grants from the National Natural Science Foundation of China (Nos. 81670470 and 81600149), a grant from the Shanghai Municipal Commission for Science and Technology (14140901600, 18411953500 and 15JC1400201) and a grant from National Key Research and Development Program (2016YFC0905100).

L. Yang, M. Liu and D. Li designed the experiments; L. Yang, $X$. Zhang, L. Wang, S. Yin, B. Zhu, L. Xie, Q. Duan, H. Han, L. Peng, Y. Wei, M. Liu, J. Zhang, H. Hu, W. Qiu and H. Geng performed the experiments; L. Yang, X. Zhang, L. Wang, S. Siwko and D. Li wrote the manuscript. D. Li supervised the research.
The authors have submitted a patent application (Application Number 2018101425473) based on the results reported in this study.

Lei Yang ${ }^{1}$, Xiaohui Zhang ${ }^{1}$, Liren Wang ${ }^{1}$, Shuming Yin ${ }^{1}$, Biyun Zhu ${ }^{1}$, Ling $\mathrm{Xie}^{1}$, Qiuhui Duan ${ }^{1}$, Huiqiong $\mathrm{Hu}^{1,2}$, Rui Zheng ${ }^{3}$, Yu Wei ${ }^{1}$, Liangyue Peng ${ }^{1,4}$, Honghui Han ${ }^{5}$, Jiqin Zhang ${ }^{1}$, Wenjuan Qiu $^{3}$, Hongquan Geng ${ }^{3}$, Stefan Siwko ${ }^{6}$, Xueli Zhang ${ }^{1,2}$, Mingyao Liu $^{1,6}$, Dali $\mathrm{Li}^{1 凶}$

${ }^{1}$ East China Normal University and Shanghai Fengxian District Central Hospital Joint Center for Translational Medicine, Shanghai Key Laboratory of Regulatory Biology, School of Life Sciences, East China Normal University, Shanghai 200241, China

2 Fengxian Hospital Affiliated to Southern Medical University, Shanghai 201499, China

${ }^{3}$ Xinhua Hospital, Shanghai Jiao Tong University School of Medicine, Shanghai 200092, China

${ }^{4}$ School of Life Sciences, Hunan Normal University, Changsha 410081, China

${ }^{5}$ Bioray Laboratories Inc., Shanghai 200241, China

${ }^{6}$ Department of Molecular and Cellular Medicine, The Institute of Biosciences and Technology, Texas AM University Health Science Center, Houston, TX 77030, USA

$\triangle$ Correspondence: dlli@bio.ecnu.edu.cn (D. Li)

\section{OPEN ACCESS}

This article is distributed under the terms of the Creative Commons Attribution 4.0 International License (http://creativecommons.org/ licenses/by/4.0/), which permits unrestricted use, distribution, and reproduction in any medium, provided you give appropriate credit to the original author(s) and the source, provide a link to the Creative Commons license, and indicate if changes were made.

\section{REFERENCES}

Frischmeyer PA, van Hoof A, O'Donnell K, Guerrerio AL, Parker R, Dietz HC (2002) An mRNA surveillance mechanism that eliminates transcripts lacking termination codons. Science 295:22582261. https://doi.org/10.1126/science.1067338

Gaudelli NM, Komor AC, Rees HA, Packer MS, Badran AH, Bryson DI, Liu DR (2017) Programmable base editing of $A \cdot T$ to $G \cdot C$ in genomic DNA without DNA cleavage. Nature 551:464-471. https://doi.org/10.1038/nature24644

Hendel A, Bak RO, Clark JT, Kennedy AB, Ryan DE, Roy S, Steinfeld I, Lunstad BD, Kaiser RJ, Wilkens AB et al (2015) Chemically modified guide RNAs enhance CRISPR-Cas genome editing in human primary cells. Nat Biotechnol 33:985-989. https://doi.org/10.1038/nbt.3290

Keeling KM, Xue X, Gunn G, Bedwell DM (2014) Therapeutics based on stop codon readthrough. Annu Rev Genomics Hum Genet 15:371-394. https://doi.org/10.1146/annurev-genom091212-153527 
Kleinstiver BP, Prew MS, Tsai SQ, Nguyen NT, Topkar VV, Zheng Z, Joung JK (2015a) Broadening the targeting range of Staphylococcus aureus CRISPR-Cas 9 by modifying PAM recognition. Nat Biotechnol 33:1293-1298. https://doi.org/10.1038/nbt.3404

Kleinstiver BP, Prew MS, Tsai SQ, Topkar VV, Nguyen NT, Zheng Z, Gonzales APW, Li Z, Peterson RT, Yeh J-RJ et al (2015b) Engineered CRISPR-Cas9 nucleases with altered PAM specificities. Nature 523:481-485. https://doi.org/10.1038/ nature14592

Komor AC, Kim YB, Packer MS, Zuris JA, Liu DR (2016) Programmable editing of a target base in genomic DNA without double-stranded DNA cleavage. Nature 533:420-424. https://doi. org/10.1038/nature17946

Kroos MA, Kirschner J, Gellerich FN, Hermans MMP, Van der Ploeg AT, Reuser AJJ, Korinthenberg R (2004) A case of childhood Pompe disease demonstrating phenotypic variability of $p$. Asp645Asn. Neuromuscul Disord 14:371-374. https://doi.org/ 10.1016/j.nmd.2004.02.012

Li X, Wang Y, Liu Y, Yang B, Wang X, Wei J, Lu Z, Zhang Y, Wu J, Huang $X$ et al (2018) Base editing with a Cpf1-cytidine deaminase fusion. Nat Biotechnol 36:324-327. https://doi.org/10.1038/ nbt.4102

Liu Z, Lu Z, Yang G, Yang G, Li G, Feng S, Liu Y, Li J, Yu W, Zhang Y et al (2018) Efficient generation of mouse models of human

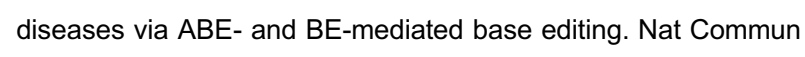
9:2338. https://doi.org/10.1038/s41467-018-04768-7

Nishida K, Arazoe T, Yachie N, Banno S, Kakimoto M, Tabata M, Mochizuki M, Miyabe A, Araki M, Hara KY et al (2016) Targeted nucleotide editing using hybrid prokaryotic and vertebrate adaptive immune systems. Science. https://doi.org/10.1126/science. aaf8729

Ryu S-M, Koo T, Kim K, Lim K, Baek G, Kim S-T, Kim HS, Kim D, Lee $H$, Chung $E$ et al (2017) Adenine base editing in mouse embryos and an adult mouse model of Duchenne muscular dystrophy. Nat Biotechnol. https://doi.org/10.1038/nbt.4148

Shao Y, Wang L, Guo N, Wang S, Yang L, Li Y, Wang M, Yin S, Han $H$, Zeng $L$ et al (2018) Cas9-nickase-mediated genome editing corrects hereditary tyrosinemia in rats. J Biol Chem. https://doi. org/10.1074/jbc.RA117.000347

Wang H, La Russa M, Qi LS (2016) CRISPR/Cas9 in genome editing and beyond. Annu Rev Biochem. https://doi.org/10.1146/ annurev-biochem-060815-014607

Zhou C, Zhang M, Wei Y, Sun Y, Sun Y, Pan H, Yao N, Zhong W, Li $\mathrm{Y}$, Li W et al (2017) Highly efficient base editing in human tripronuclear zygotes. Protein Cell 8(10):772-775
Lei Yang, Xiaohui Zhang, Liren Wang, and Shuming Yin have contributed equally to this work.

Electronic supplementary material The online version of this article (https://doi.org/10.1007/s13238-018-0568-x) contains supplementary material, which is available to authorized users. 